\title{
Connection between mathematical and visual approaches in engineering classes
}

\section{Dr. Pyoyoon Hong P.E., Southern Polytechnic State University}

Dr. Pyoyoon Hong is an assistant Professor in Architecture Department at Southern Polytechnic State University. Dr. Hong earned the Ph.D. in Structural Engineering from the University of Oklahoma, the M.S. in Structural Engineering from the University of Oklahoma, the M.S. in Architectural Engineering from Pusan National University in S. Korea, and the B.S. in Architectural Engineering from Pusan National University in S. Korea. 


\section{Connection between mathematical and visual approaches in structural engineering classes}

\section{Introduction}

It is essential that engineering students develop both quantitative and qualitative understanding of engineering concepts and principles. Although accuracy and reliability in solving quantitative problems is necessary, a qualitative understanding is required in applying concepts and principles to real-world problems, especially to innovative or unconventional ones. It becomes questionable whether the students have developed the adequate understanding of engineering principles if the students are not able to neither understand what underlies quantitative problem-solving procedures nor interpret the solution in physical form.

In 12 years of teaching, it was my most frustrating experience to see many bright students in my structures classes, capable of solving complicated quantitative problems, fail to answer on seemingly simple qualitative questions. Engineering students in my classes seemed to pay more attention to problem solving technique without being attentive to the underlying concepts. Traditional engineering textbooks and lectures may have been pushing students toward problemsolving more than toward conceptual understanding. When engineering principles are reduced to a series of calculation without apparent link to physical phenomena of particular interest, they become miserably boring subjects. The engineering students must actively engage in procreative mental activity coupled with interpretation of personal observation and experience in order to develop the genuine understanding of engineering concepts and theories that underlies real-world phenomena. But if engineering students remain as passive listeners in classes, such activity is rarely induced. In an effort to find balance and connection, and increase awareness of the interrelationship between quantitative and qualitative understanding in structures classes, a unique teaching methodology has been developed and used in the classes. The content, sequence and rationale are described in this paper in some detail.

Mathematical Thinking - Formulae and Equations

"Mechanics is the paradise of the mathematical sciences, because by means of it one comes to the fruits of mathematics." - Leonardo da Vinci

A mathematical approach is unquestionably the most exact, effective and economical way in engineering problem solving as well as in engineering education. Problem-solving is defined as a process used to obtain a best answer to an unknown or a decision subject to some constraints. (Mourtos 2004) In traditional engineering classes, the students' ability to comprehend engineering principles can successfully be obtained by manually solving a series of multiple engineering problems of progressive difficulty in the same fashion as most engineering textbooks are formatted. The results of this mathematical approach in engineering education seem to be straightforward, maybe even obvious. 
However, in this approach, lectures are generally conducted using calculation-intensive platforms and the role of the students in the lecture is relatively limited, and thus they often remain in a passive mode of learning throughout the classes. This factor may result in low levels of motivation, which in turn has caused poor interaction, inadequate understanding and low retention of structural principles. The bitter taste of calculations seems to hinder their active class participation which is an essential step toward conceptual understanding of engineering principles. Thus, the traditional teaching methodology of structural engineering principles seems to need some additional pedagogical consideration to make the students more attracted, motivated and remain focused. To wake up the students out of inactive mode of learning and proceed with the learning process, visual thinking is introduced as a basic catalyst to initial understanding and better memory. Various visual approaches are used before the mathematical and symbolic representation of an engineering concept. While a mathematical approach utilizes formulae and equations to define and clarify the engineering concepts, a visual approach uses graphics and models for communication. The gap between rigidly compliant mathematical thinking and formally flexible visual thinking must be correctly addressed, understood and bridged with carefully devised teaching methodology.

Visual Thinking - Ideas, Feelings and Intuition toward Comprehension Model

Visual communication, other than esoteric equations, is suggested to be promoted as a friendly solution for rigorous engineering teachers to speak to the inactive students. When structural concepts are introduced to students for the first time, the conventional mathematical approach is temporarily placed on hold. Instead, visible, interactive and even tangible (wherever is possible) approaches without 'number-manipulating' are opted to be used first to enhance the level of engagement of the students in the classes. More graphics and less 'number manipulation' is the key aspect of this paradigm. In other words, visual thinking is emphasized over mathematical thinking at this stage.

An academic real-time simulation program, physical models and 3-D graphical presentation tools, were as used to help the students to conceptually understand structural engineering principles. For an example, the interactive non-linear structural analysis and simulation software, Arcade, can be very effectively used to 'play' with the fundamental structural concepts, e.g., force, components, resultant, moment, equivalent force systems, equilibrium, and principle of transmissibility. In this session, the structural concepts are introduced without using the unfamiliar engineering terminologies or mathematical knowledge. It is widely accepted that students must learn statics before use structural analysis software (Black and Duff 1994) because they will not correctly interpret the analysis results. It is also academic common place to learn statics before dynamics. 
However, experience with this computer game-like program shows that dynamic simulation can be an extremely effective tool to visualize static equilibrium and other related structural engineering concepts. Students become to conceptually understand a static equilibrium through informed observation of what happens when it is broken - the object accelerates in the direction of the resultant of the forces acting on it. This software is potentially capable of enabling totally new modes of teaching in structural engineering because of its different approach to the user interface and to the underlying computation method. As students simply play with various examples of simulations they get to grasp the best possible feeling of fundamental structural concepts by shifting greater emphasis on visually thinking about the physical behavior of the
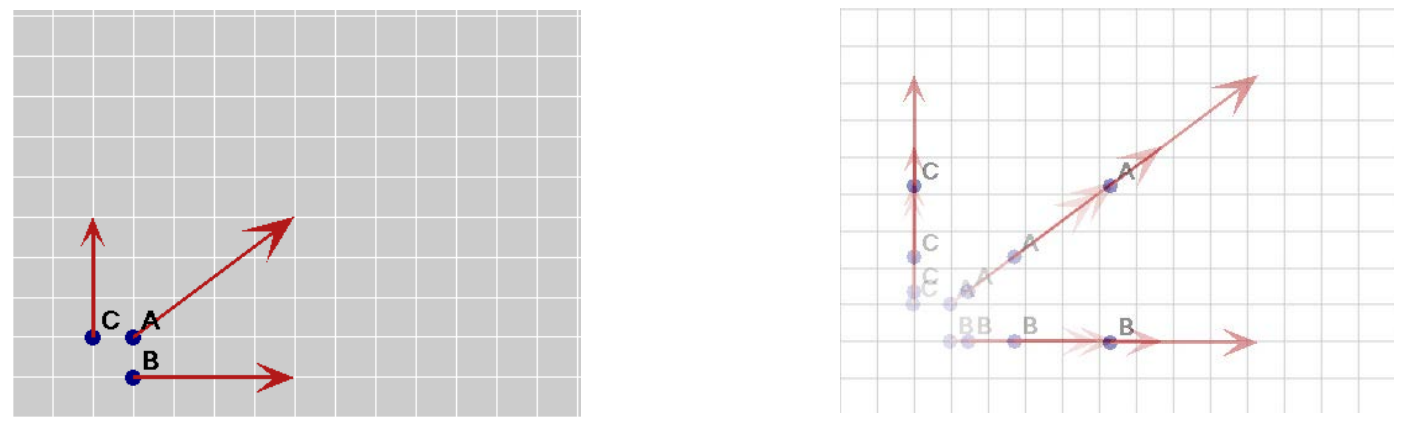

Figure 1-1 Dynamic effects of horizontal and vertical components and their resultant can be a very effective tool to acquire conceptual understanding of static equivalence of them without using trigonometry.
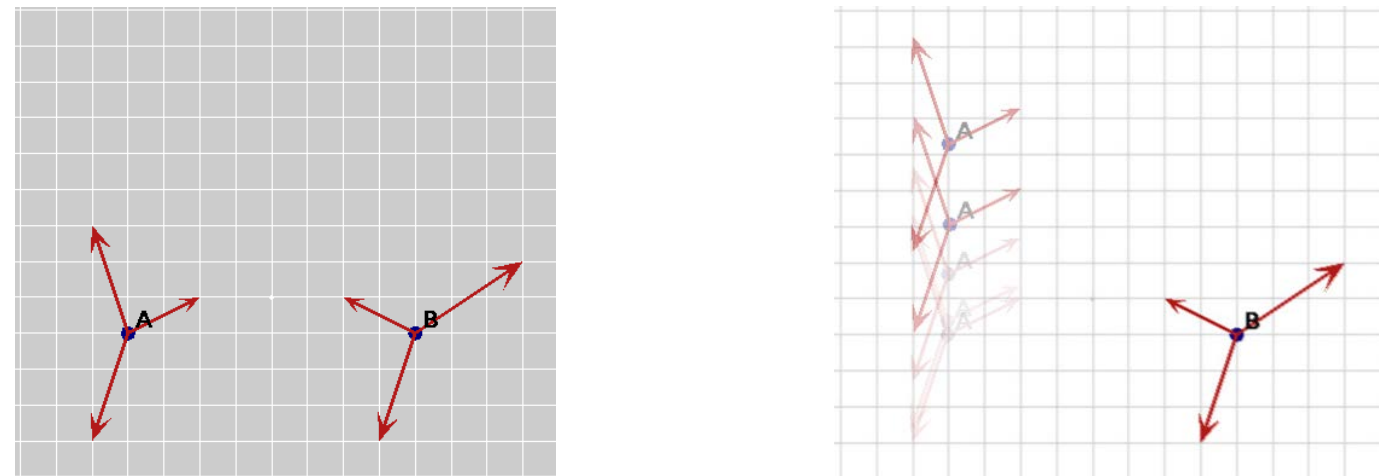

Figure 1-2 The conceptual understanding of static equilibrium can be achieved by visually analyzing the results of dynamic simulations.
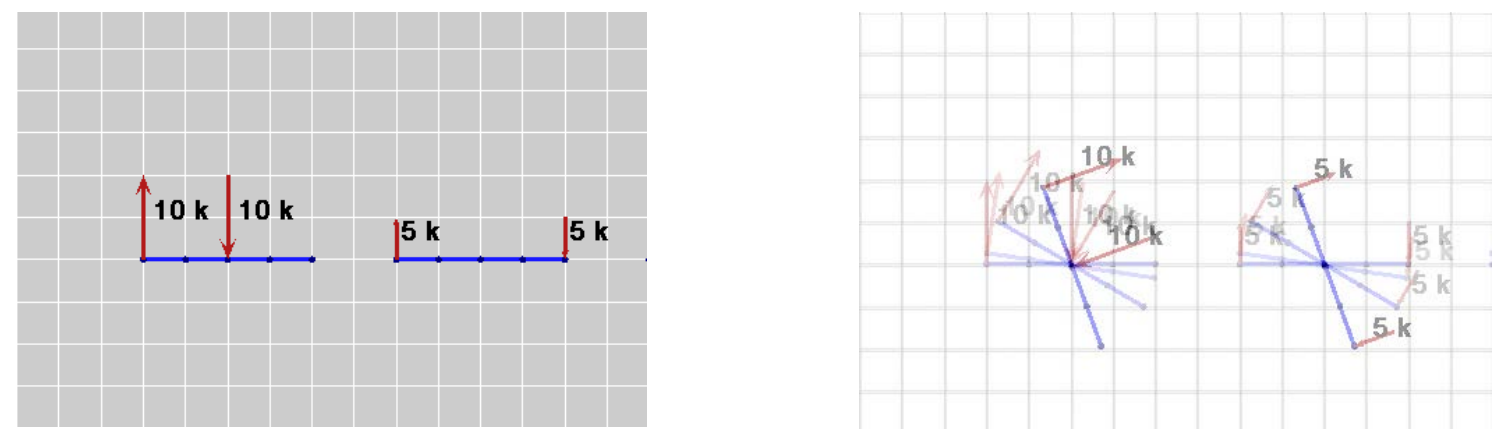

Figure 1-3 The effects of couple moments can be visually understood and quantified very easily by counting the number of unit areas that the couples enclose. 
objects with less emphasis on the mathematical thinking of the vectors. As shown in Figures 1-1 through 1-3, students begin to develop initial understanding of engineering concepts based on visual experience as they would with computer games.

A three dimensional graphics program, Sketch-up, is also intensively used in my structures classes not only to give students initial understanding but also to link mathematical thinking to visual thinking. A mathematical solution in engineering world often requires simplifying a three dimensional phenomenon to a two dimensional model. The simplified formula is easier for students to use mathematically but is at times more difficult to visualize the real-world physical behavior. As a matter of fact, I have noticed some top-standing students who are capable of solving mathematically complicated problems fail to link the numeric answer to the applicable engineering situation. Many students seem to concentrate on problem solving recipes without being attentive to the underlying concepts. Experience shows that a proper use of dynamic, interactive and three dimensional representations of engineering concepts, as in Figures 2-1 through 2-3, is essential in fundamental and conceptual understanding of the real-world phenomenon and enables paring mathematical and visual approaches very effectively.

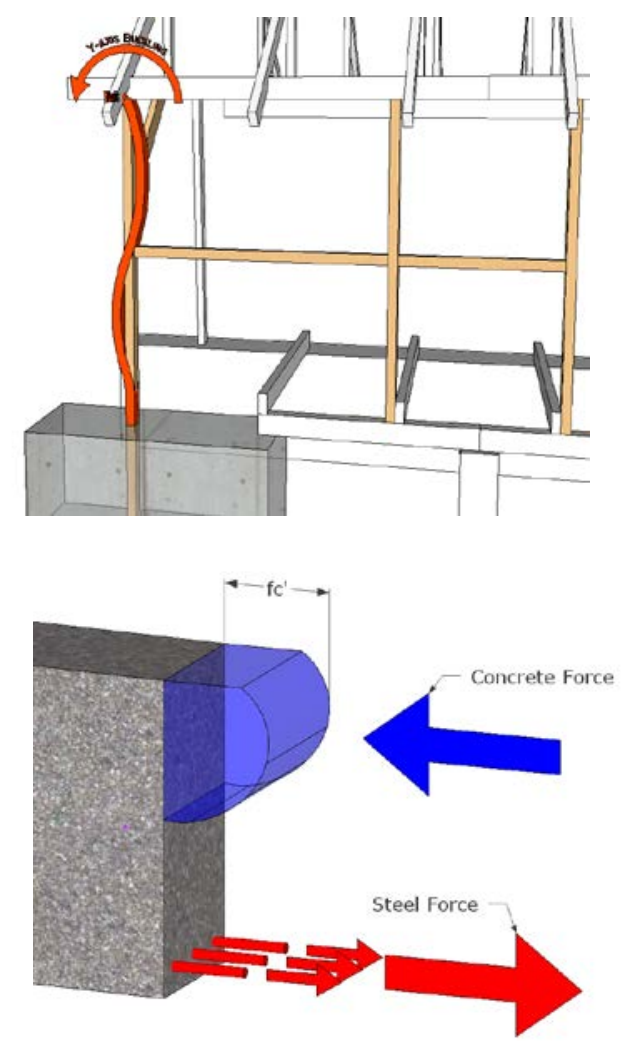

Figure 2-1 Column buckling behavior may become an unnecessarily complicated physical phenomenon to conceive without a proper 3-D interactive demonstrator because of its out-of-plane deformations.

Figure 2-2 Ultimate behavior of reinforced concrete beams and the resulting stress block can be easily explained using 3-dimensional graphics software. The magnitudes and locations of steel and concrete forces are visualized very effectively. 

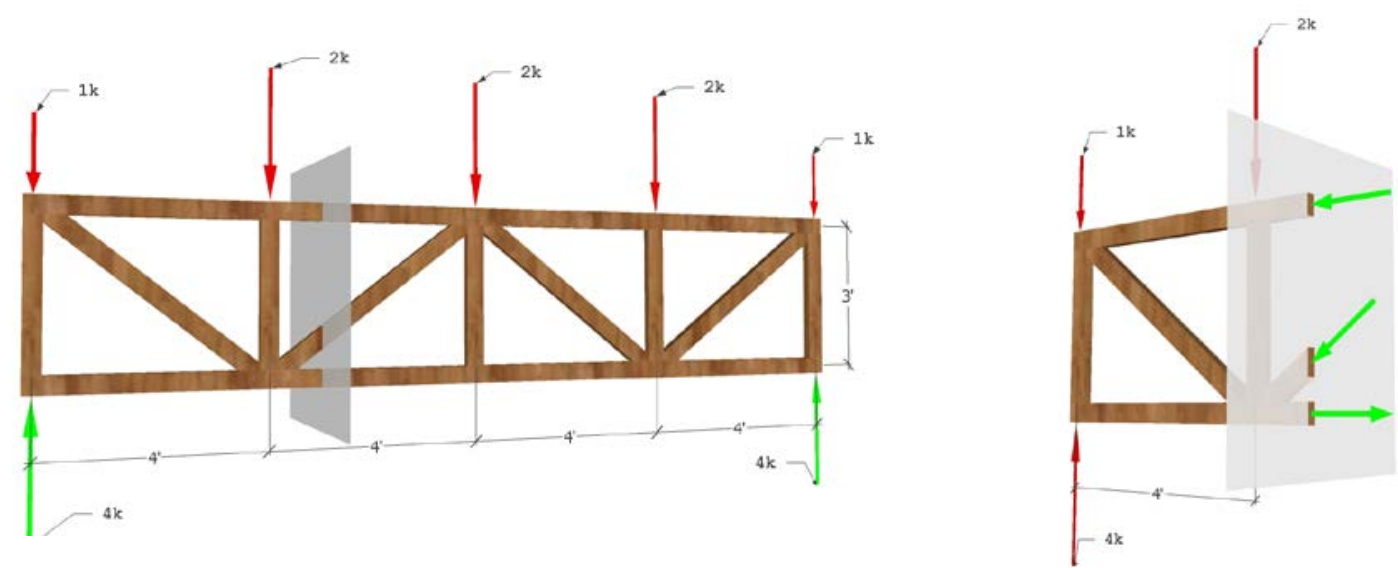

Figure 2-3 Teaching the concept of the free-body could be very challenging but this task can be easily accomplished by viewing the structural system and its free-body hanging in the air under the action of the external and the resulting internal forces that are in equilibrium.

In addition, the Force Polygons for concurrent force systems and Maxwell's diagram for analysis of determinate trusses is adopted to understand the general characteristics of truss behavior and to visualize resulting member forces. Rigorous analysis and solution are not the goals of these sessions. Rather, the sense of the forces and their approximate magnitudes, which are graphically determined with the direction and the length of the arrows, are of main concern. For the same pedagogic reason, the semi-graphical method for the shear force and bending moment diagrams are introduced followed by the equilibrium method in the later step.

\section{Bridging Visual Thinking and Mathematical Thinking - for Deeper Insight}

It is an important turning point to uncover underlying engineering principles behind the initial understanding developed through reasonable and logical 'feelings' from the previous stage. Conceptually understanding the underlying structural principles provides young engineers with a stable guideline toward feasible solutions to versatile engineering problems. To develop and nurture this intuitive talent, it is critical to train engineering students to be able to view physical phenomena from different perspective. The students are encouraged to view the physical phenomena through new engineering eyes of their own. As in Figures 3-1 through 3-3, familiar examples that can be found often around us or that one can recall easily are selected for this stage of teaching. This procedure allows the students to obtain their own viewpoint to 'read' physical phenomena and thus make appropriate links to underlying engineering concepts. In this approach, the students' ability to visualize the engineering concepts is formed and matured gradually as they try to find answers to simple qualitative problems pertaining to the engineering concept. If this training is successful, the students are prepared to start to think in structurally logical ways. 

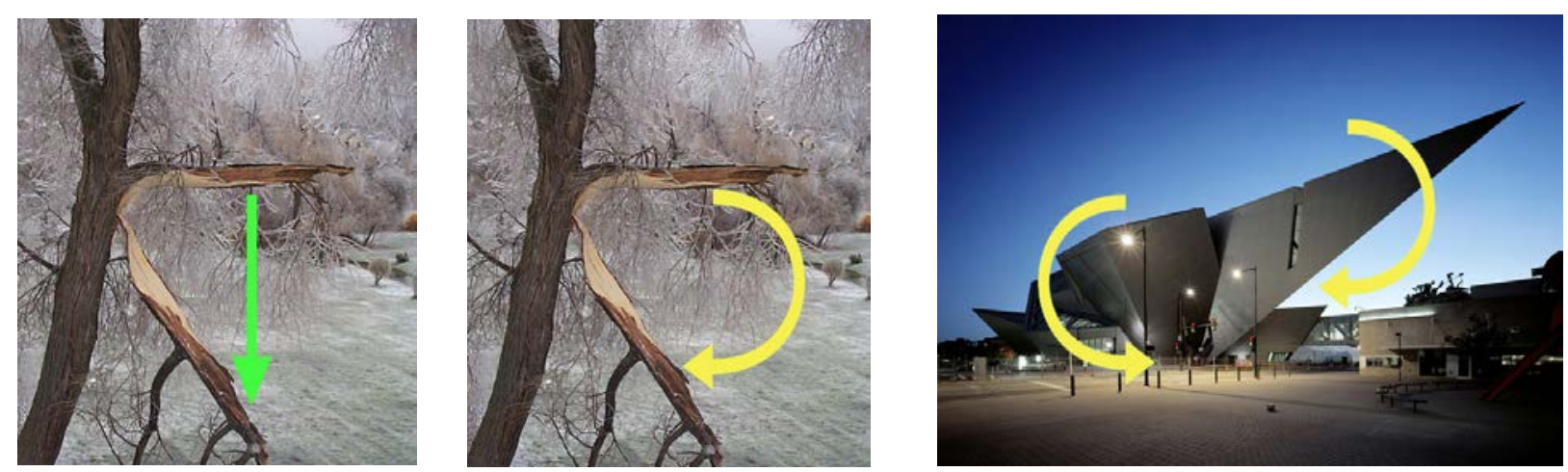

Figure 3-1 The students are instructed to view the limb as being "rotated" about the junction instead of falling "vertically" down. As a result, structural eyes to "read" the rotational equilibrium in architectural forms are properly developed.
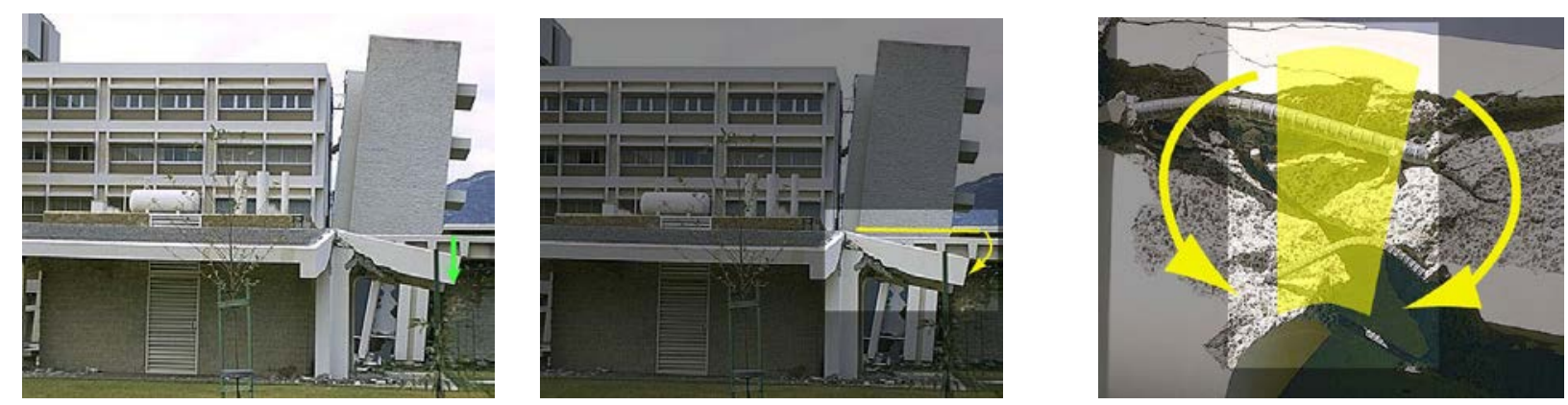

Figure 3-2 The bending failure of the canopy due to seismic tremor can better be understood by changing viewpoints.

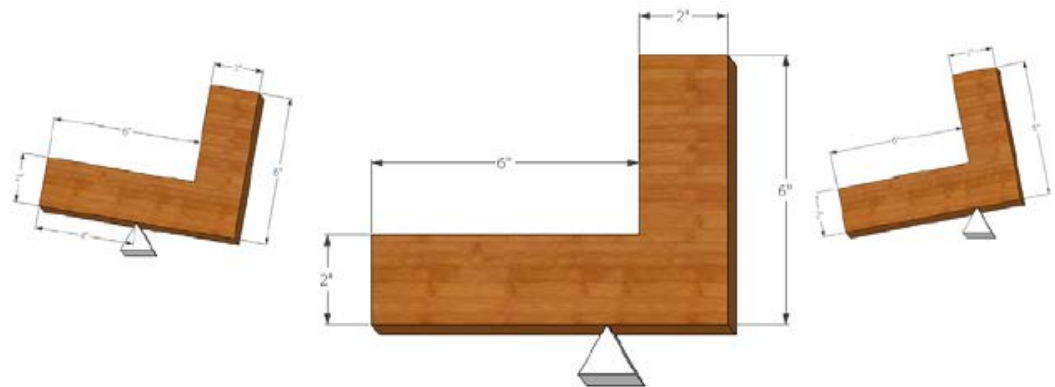

Figure 3-3 Conceptual understanding of the location of the centroidal axis can be easily obtained by changing viewpoints.

After some level of acquaintance is formed through multiple forms of visualization e.g., graphics, drawings, diagrams and demonstrations, the mathematical approach is eventually introduced. If this approach is successful, the structural engineering design formulae and related equations are not dull, life-less collections of variables and numbers. They become simple but precise textual translations of the physical phenomena as the students have viewed through their changed viewpoints. The students are expected to shift from visual thinking to mathematical thinking for mastery of knowledge. Their ability to visualize structural principles is further clarified and 
sharpened by using the engineering formulae. Instead of using structural engineering terminologies that may sound esoteric at first, the use of everyday language may be a good guideline for this stage. The link between the two may be made in the next stage more easily as the students will have become much more familiar with the abstract engineering concepts.

Some engineering formulae look complicated and some students fail to focus on how they are related to real-world phenomena. One way of keeping the students focused during the introduction of engineering formula is a sensitivity test of variables in the formula as in Figure 41. This is to monitor the change in the independent variable while the dependent variables are changed to meaningful quantities. This monitoring is made through a parallel viewing of 3dimensional interactive demonstration of the physical phenomenon and graphical presentations of the engineering formula with different font sizes of the variables under consideration. In this way, the students get to know what each engineering variable represents in reality and how sensitively it affects the final solution. During this process, the variables are no longer inactive ingredients but become dynamic and imperative elements. Sketch-up again is actively utilized for better visualization of the engineering concepts that the design formulae carry.

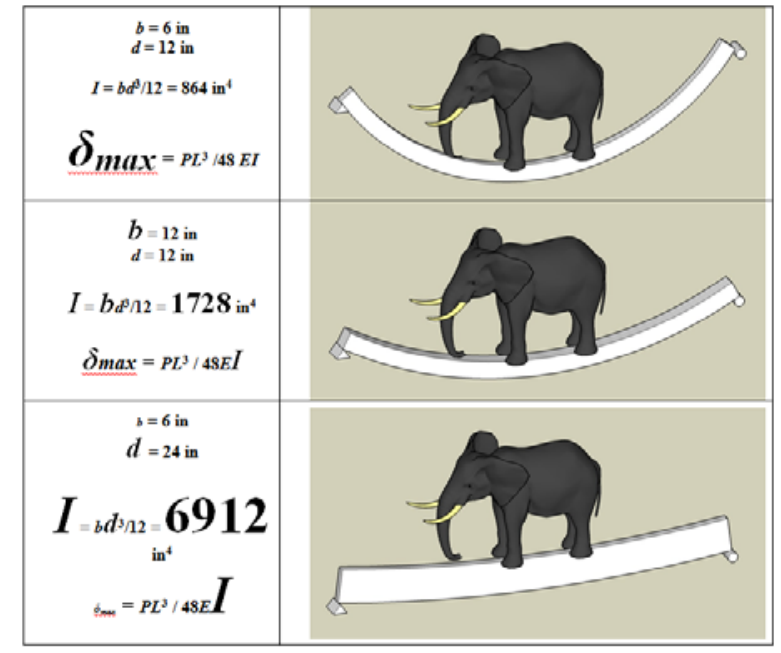

Figure 4-1 Sensitivity test of an engineering formula with different font size is used to link the variables to the real-world meaning resulting in a better retention.

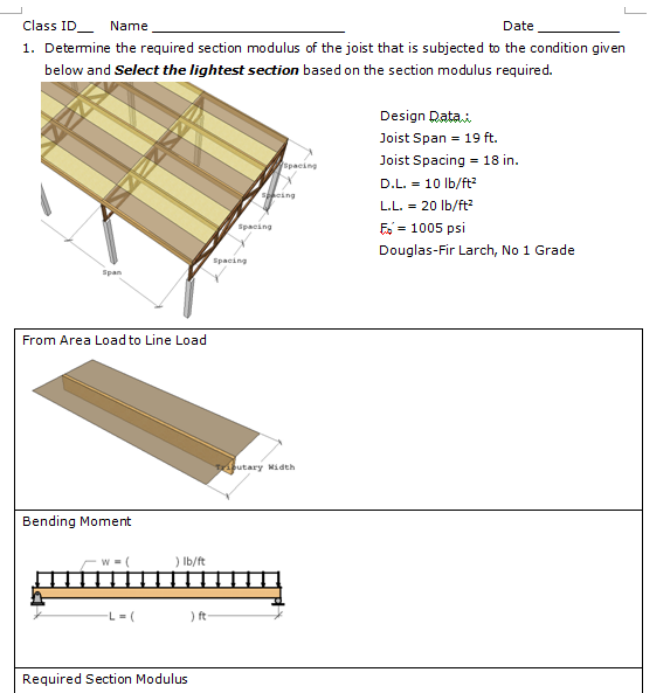

Figure 4-2 Pre-formatted case study problems are used with the same graphics used in visual approaches.

And then, the intensive mathematical approaches are eventually introduced much more actively because it is widely accepted that mathematically testing a comprehension model is the most exact, effective and economical. By now, the students are expected to have become more comfortable with the structural concepts and thus confident in their capabilities. First, a series of case studies where the structural principle under consideration is built into a reality are presented in a preformatted class notes as in Figure 4-2. In these presentations, students get to connect the 
seemingly abstract and unfriendly structural principles to familiar and aspiring built realities. So, the engineering terminologies, for which everyday language were temporarily used as intermediate steps, are eventually introduced and linked to the structural concepts that the students have obtained by feelings and ideas. A select set of structural engineering problems with progressive difficulties are utilized as lab exercises. These are almost the same as traditional engineering classes except that the same pre-formatted form is used as a guideline as in the corresponding case study. Wherever is possible, three dimensional representation of the structural concept of each step is provided in the class handout. In this stage, quantity of practice is critical for a fuller understanding and mastery of structural principles. The same graphics that were used in visual approach are inserted as illustration in quantitative case studies and lab problems to help students to link visual and mathematical understanding. While several variations of a structural formula are utilized for deeper confidence in case studies, multiple different exercises using the same formula are provided in lab problems for wider adaptability and longer retention later.

\section{Transition from Mathematical back to Visual Approaches}

At final stage, qualitative approaches are used again to explain findings from qualitative, mathematical approaches. For an example, difficult mathematical problems are paired with simple qualitative questions of the same structural concept in a qualitative quiz show contest "Weakest Link gives Jeopardy". This quiz show presentation is used again in the final review session of the semester. This teaching methodology culminates in linking structural forms to underlying structural principles in reality. Students are asked to connect bending moment diagrams with structural shapes and encouraged to be able to explain the rationale behind the structures that are found around us. An educational structural analysis software with a graphics user interface, MDSolids student version, is used to facilitate discussions through visual and oral communication rather than mathematical computation as shown in Figures 5-1 and 5-2.

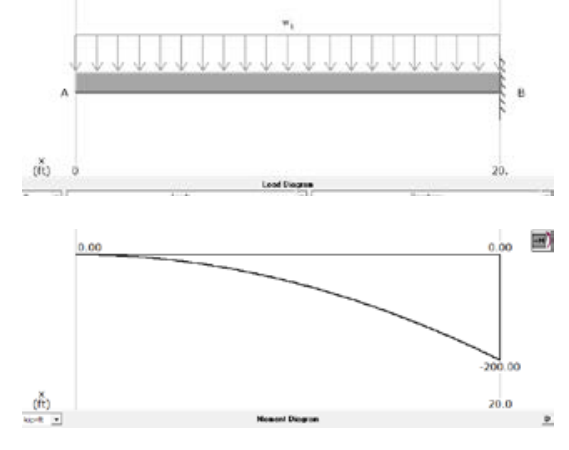

Figure 5-1 The bending moment diagram of a cantilever subjected to a uniformly distributed load.

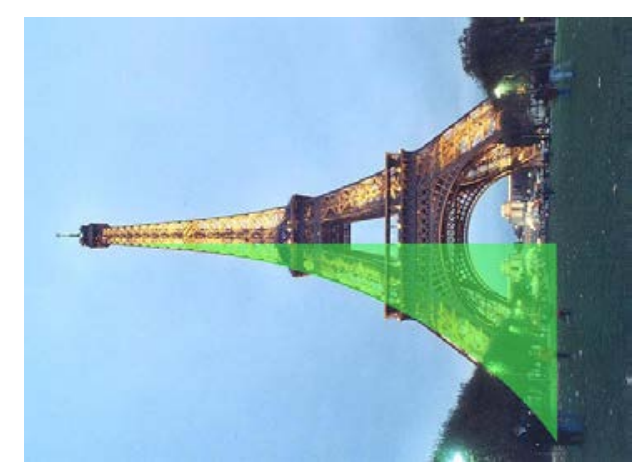

Figure 5-2 A high-riser can be viewed as a cantilever sticking out of the ground and subjected to lateral loads. 
Teaching engineering problem-solving extensively includes 'close-ended' questions that usually have unique answers and the procedure of obtaining the answer is limited or straight-forward. To simulate students' creative thinking, 'open-ended' questions must be included in engineering education. Open-ended problems address considerably the student outcomes on an ability to recognize, formulate and solve civil engineering problems and an ability to engage in lifelong learning. (Sobek and Jain, 2004) To stimulate creative thinking through open-ended problem solving skills and to verify and fortify the structural knowledge acquired through graphics and calculations, students are to work with 3-dimensional real objects in a small group environment. As shown in Figure 5-1, small-scale models, commercial construction toys, and structural term projects are utilized in this stage. The real 3-dimensional structural behavior appreciated during their project provides the students with ample opportunities to test their comprehension model and to confirm their conceptual and mathematical understanding on structural shapes and systems. Moreover, the 3-dimensional structural behaviors often reveal the limited scope of the class and allow the students to realize the gap between the textbook solutions, which are generally in 2-dimensional planes, and the real-world physical phenomenon. Brief qualitative solutions are given by the instructor about the deviation to quench academic curiosity.
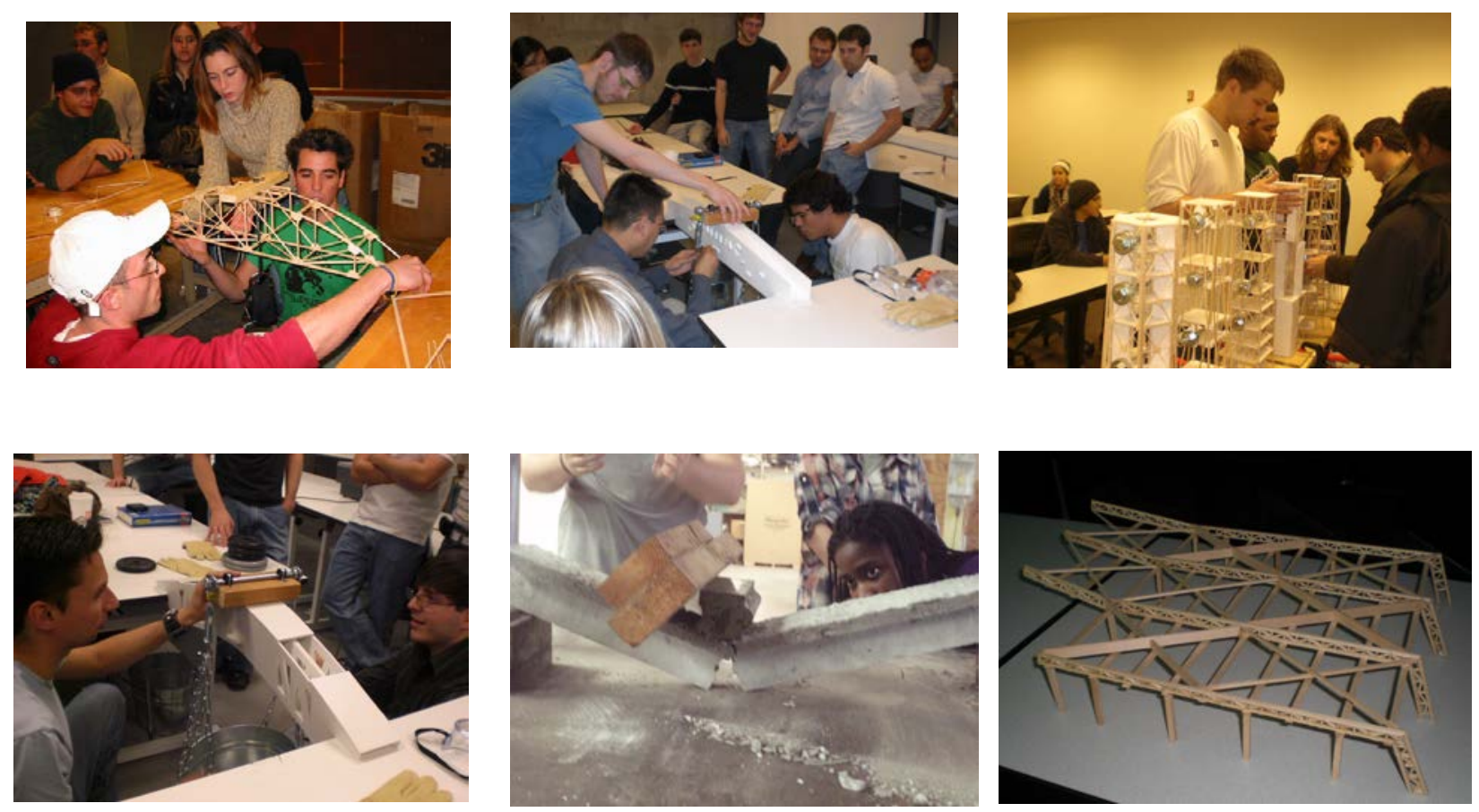

Figure 6-1 Small-scale modeling, balsa wood truss contests and plate girder contests were found to be the most challenging and yet enjoyable. These components have received the strongest supports from the students in the evaluations and surveys. The experimental performance data and team presentation material have been documented every semester for future references. 


\section{Specifics of Class Structure}

The computer software packages (Arcade, SketchUp, MDSolids and Beam Express) used for the class are all free-ware and can be installed to students' laptops with Windows operating systems without major compatibility issues. All these programs has extremely easy and convenient graphics user interface systems so a couple of demonstrations enables the students ready to use them for exercise problems with progressive difficulties. Three students are grouped together, share one computer and discuss the exercise problems and the case study problems. After the introductory sessions, students are required to keep using the computer programs for homework problems and the term project. Whenever is appropriate, the instructor use the software for demonstration as a part of presentations of new engineering concepts. In this fashion, considerably additional class hours don't have to be spent for this approach. According to my experience, 3-50 minute sessions are necessary to have the students get familiar with the software packages. SketchUp is mainly used for the instructor's dynamic, interactive, 3dimensional presentations to accommodate students' needs for various viewpoint changes. Time taken to prepare the presentation materials can be significant and depends on the number and nature of subjects to present as well as the instructor's skill of using SketchUp.

\section{Student Evaluation and Surveys}

For the last 4 years, comparison of the exam scores of the students in the 4 different sections (average enrollment of 20) of the same course in the University indicates that the students in the section with the combined visual and mathematical approaches performs 5 to $10 \%$ better than the ones in the sections with mathematical approach only. Reviews received over the last 4 years reveal that students (average age of 19-20, with a male-female student ratio of roughly 70:30) give strong approval for these approaches. Another sign of the students' support is that the enrollment for this class, among 4 sections of the same course, becomes full on the very first day of registration while the numbers of enrollment of the other sections still remain low until the last day of registration. The term project and small-scale models have received the most favorable feedback. Construction toys and physical demonstrators rank the second, the 3-D interactive demonstration tool the third, the real-time simulation sessions the fourth and the conventional mathematical approach sessions the last.

\section{Conclusions:}

1. Visual approaches can be successfully designed into structural engineering classes to provide initial understanding and better retention of the meaning of the numbers used in mathematical approach. 
2. The use of dynamic simulation can be a very effective tool to provide engineering student with fundamental and conceptual understanding of statics.

3. 'Playing' with engineering concepts without the bitter taste of mathematics more easily engages the students in the subject concepts for a deeper understanding.

4. The balance between mathematical and visual approaches in structure classes must be properly addressed and maintained since they complement each other.

5. Small-scale modeling provides students with opportunities to find that learning structures could be an enjoyable experience that leaves a stronger impression for longer retention.

6. Materialized abstract concepts in reality greatly help students to complete a comprehension model of those concepts. Studying historic and modern buildings which have distinctive structural elements as architectural expressions strongly connects them to the technological side of architecture.

7. Visualizing and experiencing 3-D structural behaviors help students realize the deviation between the textbook solutions and the real-world physical phenomena.

\section{References:}

Mourtos, N. et al. (2004). "Open-ended problem solving skills in thermal-fluids engineering" Global Journal of Egg Education, UICEE

Black, R and Duff, S. (1994). "A model for teaching structures: finite element analysis in architectural education" Journal of Architectural Education 48(1): 38-55

Sobek, D and Jain V. (2004). "The Engineering Problem Solving Process: Good for Students?" Proc. 2004 American Society for Engineering Education (ASEE) Annual Conference \& Exposition 